\title{
Economic Evidence of Pneumococcal Vaccination in Older Adults: Uncertain Modelling or Competitive Tendering?
}

\author{
Livio Garattini ${ }^{1}$ - Anna Padula ${ }^{1}$ Milene Rangel Da Costa ${ }^{1,2}$
}

Published online: 30 October 2015

(C) Springer International Publishing Switzerland 2015

\section{Introduction}

Diseases caused by Streptococcus pneumonia contribute considerably to morbidity and mortality worldwide [1]. The main types of illness are grouped as bacteremic pneumococcal pneumonia (BPP) and non-bacteremic pneumococcal pneumonia (NBPP), ${ }^{1}$ the latter causing less serious manifestations $[1,2]$.

Pneumococcal vaccination for infants has been widely debated in the last decade following the launch of the new and expensive conjugate vaccines (PCVs); unlike the old and cheap 23-valent polysaccharide vaccine (PPV23), PCV vaccines are immunogenic in children under 2 years of age [3].

Lately, the debate has shifted to the extension of PCV13 (the only PCV approved for adults) to at-risk and older adults instead of PPV23, which is still adopted in many European countries for campaigning on this population target. This hot debate $[4,5]$ is fuelled by two crucial issues that are still uncertain. First, although PPV23 has been shown to be effective in older adults, the extent and especially the duration of its protection are still doubtful [6, 7]. Second, vaccination of infants with PCVs (unlike with PPV23) provides herd immunity that has an impact on serotype replacement [8] and thus prevents disease in all ages [9], elderly included.

Against this background, economic evaluations (EEs) of pneumococcal vaccines for adults published so far have

Livio Garattini

lgarattini@marionegri.it; livio.garattini@marionegri.it

1 IRCCS Institute for Pharmacological Research "Mario Negri”, 24020 Ranica, Italy

2 Faculty of Pharmacy, Federal University of Rio de Janeiro, Rio de Janeiro, Brazil had to rely on many assumptions $[4,10]$. Very recently, the CAPiTA (Community-Acquired Pneumonia Immunization Trial in Adults) study (a Dutch clinical trial supported by the manufacturer) [11] showed that PCV13 is efficacious in preventing two thirds of BPP $(75 \%)$ and around half the NBPP $(45 \%)$ cases in older adults. The Spanish CAPAMIS (Community-Acquired Pneumonia, Acute Myocardial Infarction and Stroke) cohort study [12] (funded by public authorities) confirmed that PPV23 is effective against both BPP (62\%) and NBPP (48\%) in adults $\geq 60$ years [5], although only for up to 5 years after vaccination.

Here we critically review the most recent EEs published in the EU focussed on the elderly, to assess their potential contribution to public decision making and examine whether sponsorship by manufacturers influenced the results, as happened for studies focussed on infants [13].

\section{Economic Evaluations in the EU}

We performed a literature search on the PubMed international database to select the EEs focussed on pneumococcal vaccines conducted in EU countries on older adults, published in English from July 2012 until July 2015. ${ }^{2}$ The eight studies finally

\footnotetext{
${ }^{1}$ Other acronyms often used in studies and overlapping with these two groups are, respectively, IPD (invasive pneumococcal disease) and CAP (community acquired pneumonia).

2 The search terms used were 'pneumococcal vaccine' or 'pneumococcal conjugated vaccine' or 'pneumococcal polysaccharide vaccine' combined with 'cost' or 'cost-effectiveness' or 'economic evaluation'. We retrieved 103 articles: 65 were discarded because they did not include an EE on a pneumococcal vaccine, and were clinical or epidemiological studies (35); reviews, letters or comments (22); or cost or cost-of-illness studies (8). Then, 25 studies did not concern the EU setting and five focused on children or only high-risk groups of adults.
} 
selected [14-21] came from six countries (Germany, Italy, the Netherlands, Poland, Spain and the UK) (Table 1). Five studies considered adults aged $>65$ years as the target population [15-18, 21], one $>60$ years [20], one 65-74 years [14] and the remainder analysed only the 65-year-old cohort [19]. Five studies included a cost-effectiveness analysis (CEA) [14, $15,18,20,21]$, the other three a budget-impact analysis (BIA) $[16,17,19]$; six studies took a third-party payer's viewpoint [15-19, 21], one a societal perspective [14] and the last considered both [20]. Four of the five CEAs adopted a lifetime horizon [14, 15, 20, 21], the other [18] 5 years, as did the three BIAs [16, 17, 19]. PCV13 was included in six studies as an alternative [14-19] (one of them giving an additional dose of PPV23 in a scenario [18]), PPV23 in four [15, 17, 20, 21], and so only two, both English and signed by the same authors [15, 17], compared the two vaccines directly. All studies were based on modelling and supported by the manufacturer of the vaccine of reference, six were even co-authored by company employees [14, 15, 17, 19-21].

The efficacy of the two vaccines was based on many assumptions in all analyses, except for the very recent Dutch one [14] that referred to the CAPiTA results for PCV13. The two Italian studies both used the same very optimistic estimates for PCV13 [16, 18], while the Spanish BIA was more conservative [19]; these three studies adopted the same estimate for both BPP and NBPP. The UK [15, 17] and German studies [20] (all three co-authored by the same first two authors) used the same estimates for PPV23 (very similar to the CAPiTA results), while the Polish study [21] gave lower values for both BPP and NBPP. The two UK studies $[15,17]$, the only ones that compared the two vaccines, assumed the efficacy to be the same for both BPP and NBPP.

The prices of PCV13 were much lower in the Italian [16, 18] and Spanish studies [19], probably because the authors used those awarded in tenders for infants, while the UK $[15,17]$ and Dutch studies [14] adopted the public prices; the price of PPV23 was very high in the German study [20]. The costs induced by pneumococcal meningitis and its sequelae were included in all studies focused on PPV23 $[15,17,20,21]$, but only in one on PCV13 [18]. The cost estimates of the diseases induced by $S$. pneumonia were always higher than vaccination costs-especially in the Italian CEA [18]. All CEAs and BIAs concluded that vaccines are, respectively, cost effective and cost saving; the two UK studies that compared the two vaccines concluded in favour of PPV23 (that of the sponsor) [15, 17].

\section{Policy Implications}

Our review of recent European EEs on pneumococcal vaccines for older adults confirmed the limits of these studies in contributing to public decision making, the influence of sponsorship on results being paramount in all of them.

In general, vaccines are one of the most cost-effective health interventions by definition, since they offer a relatively cheap way of preventing substantial morbidity [22, 23]. Beside efficacy, though, their safety may raise ethical issues, particularly for infants, since vaccines-unlike drugs - are given to people without the targeted diseases. However, this is less important when vaccines are used in campaigns for elderly people like in our case. Then, if the potential healthcare costs related to preventable pathologies are considerable (like for S. pneumonia), cost effectiveness in the long run and even cost savings in the short term are likely to be forecast by any kind of model. Moreover, modellers can easily obtain the results they (and their sponsors) hope for by adopting favourable values that they are free to choose at their discretion for uncertain variables.

The choice of pneumococcal vaccine for adults seems to be a paradigmatic example of a clear 'trade-off' between efficacy and costs, and any sponsored model can easily support manufacturers' marketing strategies. Although the PCV13 efficacy should be slightly higher in BPP and its effects last longer than those of PPV23, these advantages should be lessened by the serotype mutation induced by its herd immunity in countries where it is used for infants. On the other hand, without forgetting the ten extra serotypes, the much lower price seems to be the main strength of the old PPV23 and might even make a second campaign in elderly people after some years cost effective if necessary.

Of course, public health experts are in the best position to advise decision makers whether or not to extend pneumococcal vaccination to older adults. If their advice is encouraging, as it seems to be in many EU countries, there are questions regarding whether pneumococcal vaccination in older adults is economically reasonable and with what vaccine [4], but these do not necessarily have to be addressed through EEs, whose contribution in this field has been clearly scant for public decision making. A more appropriate economic approach might be to use competitive tendering, where health authorities consider the two vaccines as alternatives and choose the cheapest. Particularly in this period of economic difficulties and widespread cuts to public expenditure, this choice could help make vaccination more sustainable. Experience with the HPV vaccines in EU countries like Italy, Sweden and the UK [24] shows that prices can be steeply reduced even when competition is minimal, that is, only two manufacturers. Moreover, if health authorities do not consider the two vaccines considered for campaigning in older adults fully equivalent, a 'quality score' could be added in tender clauses so as not to consider only the best price offered. 


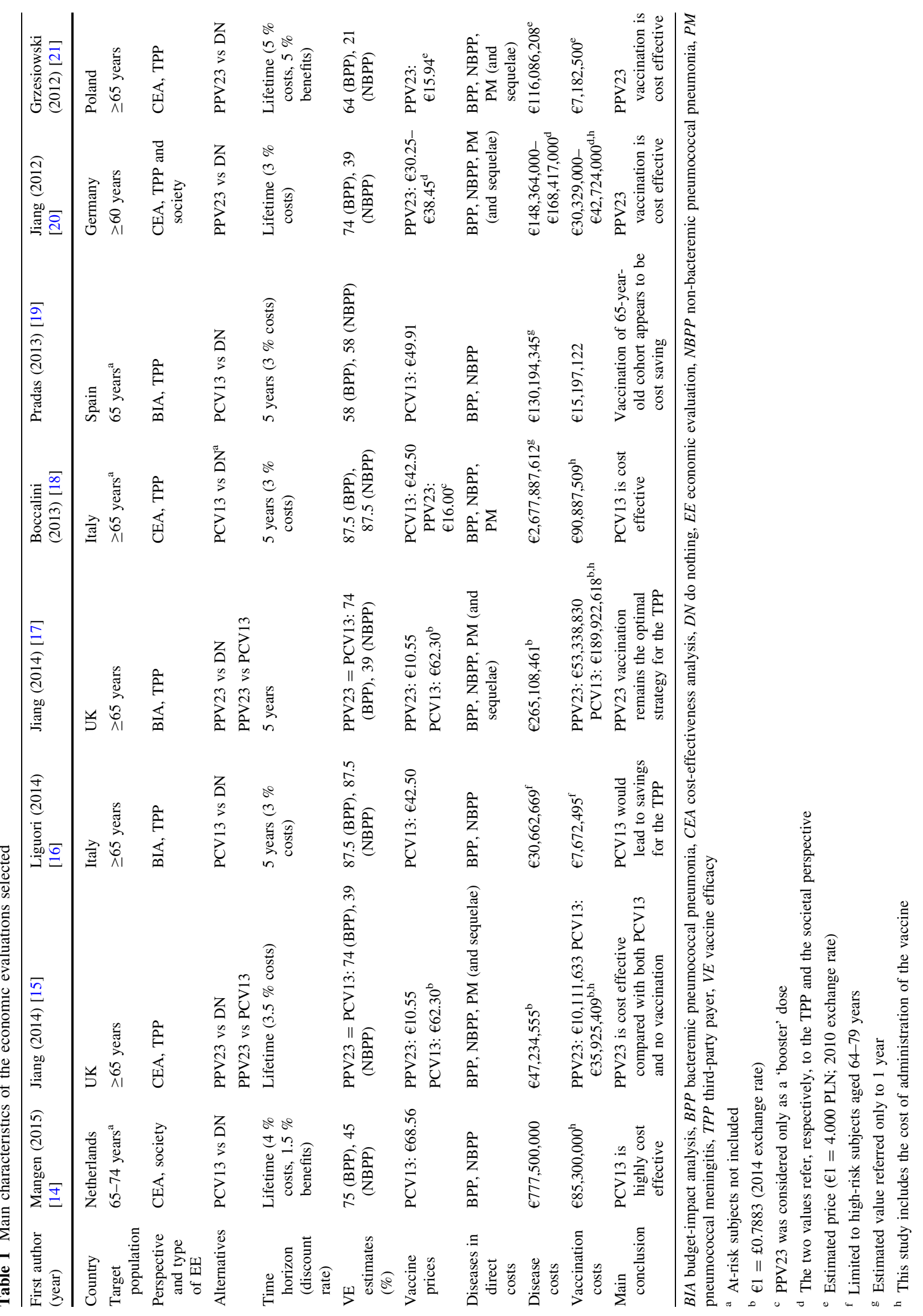


Accordingly, once it is decided that pneumococcal vaccination for older adults is a priority national health target, a reasonable economic strategy to achieve an acceptable cost-effectiveness ratio for health authorities considering the two vaccines as alternatives could be to strive for sustainable prices through competitive tendering rather than relying on uncertain models.

\section{Compliance with Ethical Standards}

Conflict of interest LG, AP and MRDC declare that they have no conflict of interest to declare.

\section{References}

1. World Health Organization. Pneumococcal disease. Immunization, vaccine and biologicals. 2015. http://www.who.int/ immunization/topics/pneumococcal_disease/en/. Accessed Aug 2015.

2. Centres for Disease Control and Prevention. About pneumococcal disease. 2015. http://www.cdc.gov/pneumococcal/about/index. html. Accessed Aug 2015.

3. Koskela M, Leinonen M, Häivä VM, et al. First and second dose antibody responses to pneumococcal polysaccharide vaccine in infants. Pediatr Infect Dis. 1986;5(1):45-50.

4. Smith KJ. Determining the cost-effectiveness of adult pneumococcal vaccination strategies. Expert Rev Pharmacoecon Outcomes Res. 2014;14(1):1-4.

5. Fedson DS. Preventing non bacteremic pneumococcal pneumonia in older adults. Hum Vaccin Immunother. 2014;10(5):1322-30.

6. Jackson LA, Neuzil KM, Yu O, et al. Effectiveness of pneumococcal polysaccharide vaccine in older adults. $\mathrm{N}$ Engl $\mathrm{J}$ Med. 2003;348(18):1747-55.

7. Huss A, Scott P, Stuck AE, et al. Efficacy of pneumococcal vaccination in adults: a meta-analysis. CMAJ. 2009;180(1):48-58.

8. Weinberger DM, Malley R, Lipsitch M. Serotype replacement in disease after pneumococcal vaccination. Lancet. 2011;378(9807):1962-73.

9. Pilishvili T, Lexau C, Farley MM, et al. Sustained reductions in invasive pneumococcal disease in the era of conjugate vaccine. J Infect Dis. 2010;201(1):32-41.

10. Dirmesropian S, Wood JG, MacIntyre CR, et al. A review of economic evaluations of 13-valent pneumococcal conjugate vaccine (PCV13) in adults and the elderly. Hum Vaccin Immunother. 2015;11(4):818-25.

11. Bonten MJ, Huijts SM, Bolkenbaas M, et al. Polysaccharide conjugate vaccine against pneumococcal pneumonia in adults. N Engl J Med. 2015;372(12):1114-25.
12. Ochoa-Gondar O, Vila-Corcoles A, Rodriguez-Blanco T, et al. Effectiveness of the 23-valent pneumococcal polysaccharide vaccine against community-acquired pneumonia in the general population aged $\geq 60$ years: 3 years of follow-up in the CAPAMIS study. Clin Infect Dis. 2014;58(7):909-17.

13. van de Vooren K, Duranti S, Curto A, et al. Cost effectiveness of the new pneumococcal vaccines: a systematic review of European studies. Pharmacoeconomics. 2014;32(1):29-45.

14. Mangen MJ, Rozenbaum MH, Huijts SM, et al. Cost-effectiveness of adult pneumococcal conjugate vaccination in the Netherlands. Eur Respir J. 2015. doi:10.1183/13993003.003252015.

15. Jiang Y, Gauthier A, Keeping S, et al. Cost-effectiveness of vaccinating the elderly and at-risk adults with the 23-valent pneumococcal polysaccharide vaccine or 13-valent pneumococcal conjugate vaccine in the UK. Expert Rev Pharmacoecon Outcomes Res. 2014;14(6):913-27.

16. Liguori G, Parlato A, Zamparelli AS, et al. Adult immunization with 13-valent pneumococcal vaccine in Campania region, South Italy: an economic evaluation. Hum Vaccin Immunother. 2014;10(2):492-7.

17. Jiang Y, Gauthier A, Keeping S. A public health and budget impact analysis of vaccinating the elderly and at-risk adults with the 23-valent pneumococcal polysaccharide vaccine or 13-valent pneumococcal conjugate vaccine in the UK. Expert Rev Pharmacoecon Outcomes Res. 2014;14(6):901-11.

18. Boccalini S, Bechini A, Levi M, et al. Cost-effectiveness of new adult pneumococcal vaccination strategies in Italy. Hum Vaccin Immunother. 2013;9(3):699-706.

19. Pradas R, Gil de Miguel A, Álvaro A, et al. Budget impact analysis of a pneumococcal vaccination programme in the 65-year-old Spanish cohort using a dynamic model. BMC Infect Dis. 2013;13:175.

20. Jiang Y, Gauthier A, Annemans L, et al. Cost-effectiveness of vaccinating adults with the 23-valent pneumococcal polysaccharide vaccine (PPV23) in Germany. Expert Rev Pharmacoecon Outcomes Res. 2012;12(5):645-60.

21. Grzesiowski P, Aguiar-Ibáñez R, Kobryń A, et al. Cost-effectiveness of polysaccharide pneumococcal vaccination in people aged 65 and above in Poland. Hum Vaccin Immunother. 2012;8(10):1382-94.

22. Kim SY, Goldie SJ. Cost-effectiveness analyses of vaccination programmes. A focused review of modelling approaches. Pharmacoeconomics. 2008;26(3):191-215.

23. Beutels P. Potential conflicts of interest in vaccine economics research: a commentary with a case study of pneumococcal conjugate vaccination. Vaccine. 2004;22(25-26):3312-22.

24. Garattini L, van de Vooren K, Curto A. Pricing human papilloma virus vaccine: lessons from Italy. Pharmacoeconomics. 2012;30(3):213-7. 